\title{
1 Title: A direct proof that sole actin dynamics drive membrane
}

\section{2 deformations}

3

\section{Camille Simon $^{\dagger 1,2}$, Rémy Kusters ${ }^{\dagger 1,2}$, Valentina Caorsi ${ }^{\dagger 1,2}$, Antoine Allard ${ }^{1,2,3}$, Majdouline} Abou-Ghali ${ }^{1,2}$, John Manzi $^{1,2}$, Aurélie Di Cicco ${ }^{1,2}$, Daniel Lévy ${ }^{1,2}$, Martin Lenz ${ }^{4}$, JeanFrançois Joanny ${ }^{1,2,5}$, Clément Campillo ${ }^{3}$, Julie Plastino ${ }^{1,2}$, Pierre Sens ${ }^{1,2 \&}$, Cécile Sykes ${ }^{1,2 \&}$

${ }^{\dagger}$ These authors contributed equally to this work.

\&These authors contributed equally to this work.

Abstract: Cell membrane deformations are crucial for proper cell function. Specialized protein assemblies initiate inward or outward membrane deformations that turn into, for example, filopodia or endocytic intermediates. Actin dynamics and actin-binding proteins are involved in this process, although their detailed role remains controversial. We show here that a dynamic, branched actin network is sufficient, in absence of any membrane-associated proteins, to initiate both inward and outward membrane deformation. With actin polymerization triggered at the membrane of liposomes, we produce inward filopodia-like structures at low tension, while outward endocytosis-like structures are robustly generated regardless of tension. Our results are reminiscent of endocytosis in mammalian cells, where actin polymerization forces are required when membrane tension is increased, and in yeast, where they are always required to overcome the opposing turgor pressure. By combining experimental observations with physical modeling, we propose a mechanism for actin-driven endocytosis under high tension or high pressure conditions.

${ }^{1}$ Laboratoire Physico Chimie Curie, Institut Curie, PSL Research University, CNRS UMR168, 75005, Paris, France. ${ }^{2}$ Sorbonne Universités, UPMC Univ Paris 06, 75005, Paris, France. ${ }^{3}$ LAMBE, Université Evry, CNRS, CEA, Université Paris-Saclay, Evry F-91025, France. ${ }^{4}$ LPTMS, CNRS, Univ. Paris-Sud, Université Paris-Saclay, 91405 Orsay, France.

${ }^{5}$ ESPCI-Paris, 10 rue Vauquelin, 75005, Paris, France. 
24 Many cell functions rely on the ability of cells to change their shape. The deformation of the cell membrane is produced by the activity of various proteins that curve the membrane inwards or outwards, by exerting pulling and pushing forces or by imposing membrane curvature via structural effects. Membrane invagination (or inward deformation of the cell membrane) can be initiated by specific proteins, such as clathrin, which coat the membrane and impose geometrical constraints that bend the membrane inwards. In this view, the action of the actin cytoskeleton, a filamentous network that forms at the membrane, is crucial only at a later stage for membrane elongation. Nevertheless, impressive correlation methods revealed unambiguously that, in yeast, membrane bending is not triggered by the presence of coat proteins, but by a dynamic actin network formed at the membrane through the Arp2/3 complex branching agent ${ }^{1-3}$. In mammalian cells, clathrin-mediated endocytosis requires the involvement of actin if the plasma membrane is tense, e.g. following osmotic swelling or mechanical stretching ${ }^{4}$. However, the exact mechanism of membrane deformation in this process is still poorly understood. Strikingly, the same type of branched actin network is able to bend the membrane the other way in dendritic filopodia, outward-pointing membrane deformations that precede the formation of dendritic spine in neurons ${ }^{5}$. Dendritic filopodia appear different from conventional filopodia where actin filaments are visibly parallel. The ability of a branched actin network to produce a filopodia-like membrane deformation has never been investigated.

44 How the same branched actin structure can be responsible for the initiation of filopodia, which are outward-pointing membrane deformations, as well as endocytic cups that deform the membrane inward, is what we want to address in this paper. Such a question is difficult to

47 investigate in cells that contain redundant mechanisms for cell deformation. Actin dynamics triggered at a liposome membrane provide a control on experimental parameters such as 
membrane composition, curvature and tension, and allow the specific role of actin dynamics

to be addressed. We evidence that the same branched actin network is able to produce both endocytosis-like and filopodia-like deformations. With a theoretical model, we predict under which conditions the stress exerted on the membrane might lead to inward and/or outward pointing membrane deformations. Combining experiments and theory allows us to decipher how the interplay between membrane tension and actin dynamics produces inward or outward membrane deformations.

\section{Membrane deformations:tubes and spikes}

Liposomes covered with an activator of the Arp2/3 complex, SpVCA, are placed in a mixture containing monomeric actin, profilin, the Arp2/3 complex and capping protein $(\mathrm{CP})$ in order to grow a branched actin network at their surface (Materials and Methods and Fig. 1A). Strikingly, imaging the membrane of liposomes in the presence of a growing actin network reveals that the liposome surface is not smooth, but instead shows a rugged profile. Indeed, membrane tubes, hereafter called "tubes", are observed to radiate from the liposome surface and extend into the actin network (Fig. 1B), even when comet formation has occurred ${ }^{6,7}$

65 (Supplementary Fig. 1). Interestingly, some liposomes display another type of membrane deformation, characterized by a conical shape that points towards the liposome interior, hereafter referred to as "spikes" (Fig. 1B). Some of the liposomes carry both tubes and spikes, while others display neither, despite the presence of an actin network at the membrane (Fig. 1B).

70 We now address the role of membrane tension on the appearance of tubes and spikes. Under conditions of normal osmotic pressure (200 mOsm), 63.0\% of liposomes display tubes only,

(Fig. 1C). To examine how membrane tension affects the occurrence of tubes and spikes, 
liposomes are deflated by increasing the osmotic pressure of the working buffer to 400 mOsm. On the one hand, a huge increase in the number of liposomes displaying spikes is observed when membrane tension is lowered in deflated liposomes. Indeed $65.0 \%$ of deflated liposomes display spikes (with or without tubes), compared to $8.4 \%$ in non-deflated conditions (Fig. 1C, p < 0.0001). On the other hand, the frequency with which tubes are observed is essentially unaffected by a change in membrane tension: $69.1 \%$ for non-deflated liposomes compared to $74.8 \%$ for deflated liposomes (not significant, $p=0.24>0.05$ ).

The presence of membrane tubes and spikes clearly correlates with the presence of the actin network. Indeed, tubes, as well as spikes, disappear where the actin network is destroyed ${ }^{6}$

(Fig. 1, D and E and Materials and Methods). Moreover, the disappearance of tubes correlates with a change in membrane aspect, from rugged to smooth (Fig. 1D). A possible effect of membrane curvature induced by our SpVCA attachment is ruled out (Supplementary Information and Supplementary Fig. 2).

\section{Characterization of tubes}

To assess where new actin monomers are incorporated during tube growth, we initiate actin assembly with Alexa568-labelled actin (red), and we incorporate new monomers of Alexa488-labelled actin (green) after 20 minutes (Materials and Methods). As previously observed for actin networks growing around polystyrene beads ${ }^{8,9}$, new monomers insert at the liposome surface (Fig. 2A). Strikingly, new (green) monomers are also observed within the already grown (red) actin network (Fig. 2A), indicating new actin incorporation on the sides of membrane tubes (evidenced by phase contrast imaging, Fig. 2A, left), where SpVCA, the activator of actin polymerization, is also present (Fig. 2B). We find that the average length of the longest tubes increases linearly with network thickness 
independent of the membrane tension (Fig. 3B, slope $0.89 \pm 0.04$ ). Moreover, we find that

100 tubes grow simultaneously with the actin network (Fig. 3, C and D and Supplementary Fig.

1013 3). An important observation is that there is a distribution of tube lengths within the actin

102 network. Indeed, shorter tubes are present, since total fluorescence intensity decreases with

103 distance from the liposome surface (Materials and Methods, Supplementary Fig. 4, A and B).

104 Tubes shorter than the network thickness are clearly visible by confocal microscopy

105 (Supplementary Fig. 4C).

106 The origin of the accumulation in membrane fluorescence detected at the tip of some of the

107 longer tubes is unclear. We observe that SpVCA forms aggregates on membranes and sticks

108 membranes together, even in the absence of actin (Supplementary Fig. 5). It is possible that

109 small vesicles are attached via SpVCA to the membrane before polymerization starts and are

110 pushed outward by actin growth. However, the presence of different tube lengths rules out

111 that tubes could be only formed by pre-existing attached vesicles.

\section{Characterization of spikes}

114 We find that new actin is incorporated at the tips of the spikes as well as at the sides (Fig.

$1154 \mathrm{~A}$ ), consistent with the localization of SpVCA (Fig. 4B). A clump of actin is observable at

116 the base of the spikes (Fig. 4C). The thickness of the clump bears no clear correlation with

117 the length of the spikes (Supplementary Fig. 6A), but slightly correlates with their width

118 (Supplementary Fig. 6B). Spikes initially elongate with time until polymerization slows

119 down, the basal width of spikes, however, remains roughly constant over time (Fig. 4D and

120 Supplementary Fig. 6C).

\section{Theoretical models for the growth of spikes and tubes}


123 To rationalize the occurrence of spike-like structures arising solely from a uniformly

124 polymerizing actin network, we use analytical modeling and numerical Finite Element

125 calculations to evaluate the conditions under which large-scale membrane deformations may

126 develop due to actin polymerization. We first estimate the normal stress exerted by the

127 polymerization of an actin network (Material and Methods for details). The actin network can

128 be modeled as a viscoelastic material with an elastic behavior at short time and a viscous

129 behavior at long time due to network rearrangement, the cross-over time being on the order of

$13010 \mathrm{~s}^{10-12}$. We choose to focus on the viscous behavior as the growth of the network occurs on

131 timescales of tens of minutes.

132 We model the growth of the actin network by imposing a uniform actin polymerization

133 velocity $v_{p}$ normal to the liposome membrane, a choice motivated by the dual color

134 measurements in Fig. 4A. We solve the Stokes equation for the viscous gel polymerizing with

135 a constant velocity normal to the liposome surface (Material and Methods). Actin

136 polymerization on a flat membrane results in a uniform actin flow which does not generate

137 any mechanical stress. A small perturbation of membrane shape modulates the actin velocity

138 field which may generate viscous stress on the membrane. We show in the Material and

139 Methods that the first order contribution to the normal stress exerted by the network on a

140 periodically weakly deformed membrane, as illustrated in Fig. 5A, also vanishes identically.

141 The lowest order contribution to the actin stress is quadratic with membrane deformation.

142 This is in agreement with the finding that actin growing on a uniformly curved surface

143 creates a normal stress proportional to the square of the curvature ${ }^{10,13}$. In the case of a

144 localized membrane perturbation, a Gaussian with amplitude $A$ and width $b$,

$145 u(x)=A \exp -(x / b)^{2}$ (Fig. 5B), we numerically calculate the normal stress exerted by an

146 actin layer (Material and Methods). We obtain the pressure and velocity fields that arise in

147 the actin layer (Fig. 5C). Velocity gradients in the growing actin layer, generated by the 
148 deformed surface, induce a normal force in the center of the perturbation, "pushing" the

149 membrane inwards in the center of the perturbation (Fig. 5D). A scaling analysis of the

150 Stokes equation, confirmed by our numerical calculation, shows that the normal stress $\sigma_{n n}$, at

151 the center of the perturbation $(x=0)$ scales as $\sigma_{n n} \sim-\eta A^{2} b^{-3} v_{p}$, where $\eta$ is the viscosity of

152 the actin layer (Fig.5, E and F). It is important to realize that the normal stress exerted by the

153 actin network on the membrane, when integrated over the area that surrounds the

154 deformation, amounts to a zero net force. This contrasts with existing models of filopodia

155 formation, which usually consider bundled actin filaments exerting a net pushing force on the

156 membrane ${ }^{14}$, invoking the friction force of treadmilling actin filament on the cellular actin

157 cortex to balance this pushing force ${ }^{15}$. While the latter approach might be appropriate to

158 explain the physics of filopodia filled with bundled actin, here, we do not a priori distinguish

159 the detailed structure of the actin network at the membrane from the one in the protrusion,

160 treating the actin network as a continuum.

161 The normal stress, on a deformable surface, is in our case balanced by the restoring elastic

162 stress $\sigma_{m e m b}$ due to membrane elasticity. Neglecting the contribution of the membrane

163 bending rigidity $\kappa$ for simplicity, this stress corresponds to the membrane Laplace

164 pressure $\sigma_{m e m b}=-\gamma C$, where $\gamma$ is the membrane tension and $C \sim A / b^{2}$ the local curvature

165 (evaluated at the center of the localized perturbation). The balance of actin polymerization

166 and membrane stresses defines a threshold amplitude $A^{*}=\gamma b /\left(\eta v_{p}\right)$. When the amplitude of

167 the perturbation is smaller than this threshold $\left(A<A^{*}\right)$ the membrane stress dominates and

168 the perturbation relaxes. Above the threshold $\left(A>A^{*}\right)$ the force exerted by the network is

169 dominant and the instability develops. For $\gamma \approx 10^{-6} \mathrm{~N} / \mathrm{m}^{16}, \eta \approx 10^{4} \mathrm{~Pa}$ s (obtained through

170 a scaling law from the elastic modulus $E$ of the network and the viscoelastic time scale

$171 \tau_{v e}$ as $\eta \approx E \tau_{v e}$, where the elastic modulus $E \approx 10^{3} \mathrm{~Pa}^{17}$ and the viscoelastic timescale

$172 \tau_{v e} \approx 10 s^{11,12}$ ) and $v_{p} \approx 10^{-9} \mathrm{~m} / \mathrm{s}$, the critical amplitude of a perturbation with a width $b \approx$ 
$17310^{-7} \mathrm{~m}$ is found to be $A^{*} \approx 10^{-8} \mathrm{~m}$. To evaluate whether such a perturbation could be

174 reached by thermal fluctuations, the average membrane roughness corresponding to the

175 fluctuation of a free membrane at thermal equilibrium is estimated as follows. With $\xi$ the

176 mesh size of the network, identifying $(A / b)^{2}$ with the thermal average of the gradient of the

177 membrane shape $\left\langle|\nabla h|^{2}>\sim \frac{k T}{2 \pi \kappa} \log \left(\frac{4 \pi^{2} \kappa}{\xi^{2} \gamma}\right){ }^{18}\right.$ and integrating over all wavelengths superior

178 to the mesh size, we find a threshold tension for the appearance of spikes: $\gamma<\gamma^{*}=$

$179 \eta v_{p} \sqrt{k T /(2 \pi \kappa)} \approx 2 \times 10^{-6} \mathrm{~N} / \mathrm{m}$. This value is in the range of membrane tension for

180 liposomes under control conditions (i.e. non-deflated liposomes), but is larger than the

181 tension of deflated liposomes, leading to the prediction that deflated liposomes are prone to

182 the formation of spikes. In agreement with our experiments, the occurrence of spikes in non-

183 deflated conditions $(8.4 \%$, Fig. $1 \mathrm{C})$ is significantly lower than in deflated conditions $(65.0 \%$,

184 Fig. 1C). A decrease of membrane tension has therefore a major influence on spike initiation.

We now develop a model for tube initiation. We consider a membrane deformation consisting depends on membrane bending rigidity $\kappa$ and membrane tension $\gamma$ through $f_{\text {tube }}=2 \pi \sqrt{2 \kappa \gamma}$, as extensively studied both theoretically and experimentally ${ }^{19,20}$. Taking the bending energy of $10 \mathrm{kT}$ and a membrane tension of $\gamma \sim 10^{-6} \mathrm{~N} / \mathrm{m}^{16}$, we find $f_{\text {tube }} \sim 2 \mathrm{pN}$. For the dynamic actin network to be able to pull a membrane tube, the tube force must be balanced by the mechanical stress in the growing network. Transient attachments between the membrane and the network exist when an actin filament is bound to the activator pVCA, as characterized

194 experimentally ${ }^{21}$. The growth of the actin network exerts a pulling force on this attachment 195 site that can pull a membrane tube. Upon detachment, the tube retracts until one of the binding sites alongside the tube reaches the tube end, thus taking turn on the extraction force 
197 (Fig. 6A). This effectively results in the network exerting a long-lived localized force on the membrane, for times longer than the viscoelastic relaxation time, supporting a viscous description of the actin layer (Supplementary Information).

The drag force exerted by the actin network (moving away from the liposome surface at a velocity $v_{p}$ ) on the tip of the tube (moving at a velocity $\dot{L}$ ) can be crudely estimated using the Stokes law: $f_{\text {drag }}=6 \pi \eta r_{\text {tube }}\left(v_{p}-\dot{L}\right)$ (Fig. 6B). At steady-state, the drag force balances the tube force, which provides the tube extraction velocity, $\dot{L}=v_{p}\left(1-\frac{f_{\text {tube }}}{6 \pi \eta r_{\text {tube }} v_{p}}\right)$. Tube extraction $(\dot{L}>0)$ is therefore possible provided

$$
\gamma \xi^{4}<\frac{3}{2} k_{B} T l_{p} v_{p} \tau_{v e}
$$

206 where we have used that $\eta \approx E \tau_{v e} \approx k_{b} T l_{p} \tau_{v e} / \xi^{422}$ (Fig. 6C). While a large enough membrane tension can in principle prevent tube extraction, the range of tension explored experimentally yields $6 \pi \eta r_{\text {tube }} v_{p}=\frac{3 \eta v_{p}}{2 \gamma} f_{\text {tube }} \approx 10 f_{\text {tube }}\left(\right.$ with $v_{p}=1 \mathrm{~nm} / \mathrm{s}, \eta=E \tau_{v e} \approx$ $10^{4} \mathrm{~Pa} . \mathrm{s}$ and $\left.\gamma \sim 10^{-6} \mathrm{~N} / \mathrm{m}\right)$. Consequently, we find that membrane tubes can always be extracted by the growing actin network under the present conditions. Tube extraction

211 however could in principle be prevented under high tension, or for a loose network (high

212 value of the actin meshsize $\xi>100 \mathrm{~nm}$ ). However, these regimes could not be explored due

213 to experimental limitations of network growth under such conditions ${ }^{23}$. Moreover the tube

214 extraction velocity is very close to the actin polymerization velocity ( $\dot{L} \gtrsim 0.9 v_{p}$ ). This

215 explains why tubes initiated early during actin growth actually span the entire actin layer. The

216 distribution in tube lengths inferred from Supplementary Fig. 4 can originate either from a

217 distribution of tube nucleation time during the growth of the network or a distribution of

218 rebinding time during tube retraction following a detachment from the actin network. 
In yeast, actin is absolutely required for endocytosis, likely because of the high turgor pressure that opposes inward membrane deformations ${ }^{24-26}$. The force needed to overcome the turgor pressure can reach $1000 \mathrm{pN}^{27}$, almost three orders of magnitude larger than the actin

223 force in our in-vitro conditions. Using actin dynamics parameters relevant for yeast

224 (polymerization velocity $v_{p}=50 \mathrm{~nm} / \mathrm{s}^{1}$ and actin network viscosity $\eta=210^{5} \mathrm{~Pa} . \mathrm{s}^{28}$ ), the drag force generated by the actin network may indeed create the force required for membrane deformation leading to endocytosis (Supplementary Information).

\section{Discussion}

The cell is a robust system where redundant mechanisms insure proper function, which makes detailed cell mechanisms difficult to decipher. This is true for membrane deformations

231 into filopodia ${ }^{5}$ or endocytic intermediates ${ }^{1}$. Here, we show that a branched actin network

232 growing at a membrane is able to mimic the initiation of either an endocytosis-like or a

233 filopodia-like deformation. Endocytosis-like deformations appear to be a robust feature with regard to membrane tension whereas the initiation of filopodia-like structures is eased by a decreased membrane tension. Our results support recent findings that the initiation of dendritic filopodia and endocytosis primarily relies on the growth of a branched actin network ${ }^{1,3,5}$.

238 Endocytosis is intimately dependent on the existence of a physical link between the actin

239 network and the plasma membrane in yeast as well as in mammalian cells under high tension.

240 Controlled endocytosis is abolished in yeast if this link is suppressed, although already

241 endocytosed vesicles retain their extraordinary capacity to polymerize actin and even undergo

242 actin-based motility ${ }^{3,29}$. In our reconstituted system, in which actin nucleators are

243 permanently linked to the liposome membrane, actin dynamics alone have the remarkable 
244 capacity to initiate endocytosis-like membrane deformations with a width smaller than, or of

245 the order of, the actin mesh size.

246 A class of model for filopodia initiation assumes a particular actin organization in the

247 protrusion, typically that of bundled actin filaments ${ }^{14,15,30,31}$. Supported by our dual color

248 actin measurements, our model for spike initiation assumes that actin polymerization occurs

249 uniformly at the membrane, which indicates that new actin is incorporated all along the

250 conical membrane surface, and not only at the tip of the protrusion as observed in Liu ${ }^{32}$.

251 Decreasing the membrane tension of the liposome decreases the critical amplitude for spike

252 nucleation and increases the likelihood of spike formation (Fig. 5). This suggests a

253 mechanism of spike formation different from that of tip growing protrusions, both in its

254 initiation, and in its subsequent growth dynamics. Spikes are mimics of filopodia, especially

255 in the case of dendritic filopodia whose formation relies on the Arp2/3 complex-branched

256 network ${ }^{5}$, as the suppression of the Arp2/3 complex system decreases the number of

257 dendritic protrusions ${ }^{33}$.

\section{Materials and Methods}

1. Reagents, lipids, proteins

261 Chemicals are purchased from Sigma Aldrich (St. Louis, MO, USA) unless specified

262 otherwise. L-alpha-phosphatidylcholine (EPC), 1,2-distearoyl-sn-glycero-3-

263 phosphoethanolamine-N-[biotinyl polyethylene glycol 2000] (biotinylated lipids), 1,2-

264 dioleoyl-sn-glycero-3- [[N(5-amino-1-carboxypentyl)iminodiacetic acid]succiny] nickel salt

265 (DOGS-NTA-Ni) are purchased from Avanti polar lipids (Alabaster, USA). Texas Red® 1,2-

266 dipalmitoyl-sn-glycero-3-phosphocholine, triethylammonium salt is from Thermofisher.

267 Actin is purchased from Cytoskeleton (Denver, USA) and used with no further purification.

268 Fluorescent Alexa-488 actin and Alexa546 actin are obtained from Molecular Probes 
269 (Eugene, Oregon, USA). Porcine Arp2/3 complex is purchased from Cytoskeleton and used 270 with no further purification. Biotin is purchased from Sigma-Aldrich (St. Louis, Missouri,

271 USA), diluted in DMSO. Mouse $\alpha 1 \beta 2$ capping protein is purified as in ${ }^{34}$. Untagged human 272 profilin and SpVCA are purified as in ${ }^{7}$. SpVCA is fluorescently labeled on the N-terminal 273 amine with Alexa546 at $\mathrm{pH} 6.5$ for $2 \mathrm{~h}$ at $4{ }^{\circ} \mathrm{C}$, desalted and then purified on a Superdex 200 274 column. His-pVCA-GST (GST-pVCA) is purified as for PRD-VCA-WAVE ${ }^{35}$ and HispVCA is essentially the same without the glutathione sepharose step. A solution of $30 \mu \mathrm{M}$ monomeric actin containing $15 \%$ of labeled Alexa- 488 actin is obtained by incubating the actin solution in G-Buffer ( $2 \mathrm{mM}$ Tris, $0.2 \mathrm{mM} \mathrm{CaCl} 2,0.2 \mathrm{mM}$ DTT, $\mathrm{pH}$ 8.0) overnight at $4^{\circ} \mathrm{C}$. All proteins (SpVCA, profilin, $\mathrm{CP}$, actin) are mixed in the isotonic or hypertonic working buffer. The isotonic working buffer contains $25 \mathrm{mM}$ imidazol, $70 \mathrm{mM}$ sucrose, 1 mM Tris, 50 mM KCl, 2 mM MgCl2, 0.1 mM DTT, 1.6 mM ATP, 0.02 mg/mL $\beta$-casein, adjusted to $\mathrm{pH}$ 7.4. The hypertonic working buffer differs only by its sucrose concentration and contains $25 \mathrm{mM}$ imidazol, $320 \mathrm{mM}$ sucrose, $1 \mathrm{mM}$ Tris, $50 \mathrm{mM} \mathrm{KCl,} 2 \mathrm{mM} \mathrm{MgCl} 2,0.1$ mM DTT, $1.6 \mathrm{mM}$ ATP, $0.02 \mathrm{mg} / \mathrm{mL} \beta$-casein, adjusted to $\mathrm{pH} 7.4$. Osmolarities of the isotonic and hypertonic working buffers are respectively 200 and 400 mosmol, as measured with a Vapor Pressure Osmometer (VAPRO 5600). In case of experiments with DOGSNTA-Ni lipids, all proteins are diluted in a working buffer containing $280 \mathrm{mM}$ glucose, 10 mM HEPES, 0.5 mM DABCO, $100 \mathrm{mM} \mathrm{KCl,} 4 \mathrm{mM} \mathrm{MgCl} 2,1 \mathrm{mM}$ DTT, $10 \mathrm{mM}$ ATP and $0.05 \mathrm{mg} / \mathrm{mL} \beta$-casein.

\section{Liposome preparation}

291 Liposomes are prepared using the electroformation technique. Briefly, $10 \mu \mathrm{l}$ of a mixture of

292 EPC lipids, 0.1\% biotinylated lipids or 5\% DOGS-NTA-Ni lipids, and 0.1\% TexasRed lipids 293 with a concentration of $2.5 \mathrm{mg} / \mathrm{ml}$ in chloroform $/$ methanol 5:3 (v/v) are spread onto indium 
294 tin oxide (ITO)-coated plates under vacuum for $2 \mathrm{~h}$. A chamber is formed using the ITO

295 plates (their conductive sides facing each other) filled with a sucrose buffer ( $0.2 \mathrm{M}$ sucrose, 2

296 mM Tris adjusted at pH 7.4) and sealed with hematocrit paste (Vitrex Medical, Denmark).

297 Liposomes are formed by applying an alternate current voltage $(10 \mathrm{~Hz}, 1 \mathrm{~V})$ for $2 \mathrm{~h}$.

298 Liposomes are then incubated with an activator of actin polymerization (SpVCA, $350 \mathrm{nM})$

299 via a streptavidin-biotin link for $15 \mathrm{~min}$. Isotonic liposomes are used right away for

300 polymerizing actin in the isotonic working buffer. To obtain deflated liposomes, an extra step

301 is added: they are diluted twice in the hypertonic working buffer at $400 \mathrm{mOsmol}$ and

302 incubated for $30 \mathrm{~min}$. The final solution is therefore at $300 \mathrm{mOsmol}$.

\section{2bis. Biotin-blocking experiments}

304 SpVCA labeled with AlexaFluor546 and biotin are diluted in the isotonic working buffer and incubated for 10 min to reach final concentration of $350 \mathrm{nM} \mathrm{SpVCA}$ and various concentrations of biotin $(87.5 \mathrm{nM}, 175 \mathrm{nM}, 262.5 \mathrm{nM}, 350 \mathrm{nM})$. Note that $350 \mathrm{nM}$ of biotin (99.9\% EPC lipids, $0.1 \%$ biotinylated lipids) are then diluted twice in this solution and incubated for $15 \mathrm{~min}$. Tubes and spikes are visualized by the fluorescence of SpVCA.

\section{Actin cortices with a branched network}

312 Condition 1: Actin polymerization is triggered by diluting the isotonic or deflated liposomes

3136 times in a mix of respectively isotonic or hypertonic working buffer containing final

314 concentrations of $3 \mu \mathrm{M}$ monomeric actin (15\% fluorescently labelled with AlexaFluor488), 3

$315 \mu \mathrm{M}$ profilin, $37 \mathrm{nM}$ Arp2/3 complex, $25 \mathrm{nM} \mathrm{CP}$. Note that the final concentrations of salt and

316 ATP in both isotonic and hypertonic conditions are $0.3 \mathrm{mM} \mathrm{NaCl}, 41 \mathrm{mM} \mathrm{KCl}, 1.6 \mathrm{mM}$

$317 \mathrm{MgCl} 2,0.02 \mathrm{mM} \mathrm{CaCl} 2$ and $1.5 \mathrm{mM}$ ATP. Hypertonic conditions differ from isotonic

318 conditions by $250 \mathrm{mM}$ sucrose. 
319 Condition 2: Same protocol as in Condition 1 with unlabeled monomeric actin, unlabeled

320 liposomes (99.9\% EPC lipids, 0.1\% biotinylated lipids) and SpVCA labeled with

$321 \quad$ AlexaFluor546.

322 In Figure 1, panel C, non-deflated liposomes n=311 are distributed as follows: 215 from 3

323 experiments in Condition 1 and 96 from 2 experiments in Condition 2. Deflated liposomes $\mathrm{n}=123$

324 are distributed as follows: 92 from 2 experiments in Condition 1 and 31 from one experiment 325 in Condition 2.

\section{Photo-damage of the actin network}

328 The actin network area to photo-damage is defined with a diaphragm. The area is illuminated 329 for $15 \mathrm{~s}$ with a Hg lamp and a FITC filter cube and the illumination is repeated until actin is completely destroyed or at least no longer detectable by eye.

\section{Two color experiment}

333 Liposomes are first incubated with $350 \mathrm{nM}$ SpVCA for $15 \mathrm{~min}$. This solution is then diluted

3343 -fold into a mix of isotonic buffer containing $3 \mu \mathrm{M}$ actin (15\% Alexa568-labeled, red), 37 nM Arp2/3 complex and $25 \mathrm{nM} \mathrm{CP}$. After 20 min of incubation in these conditions, the solution is diluted 3 times in a mix of same protein concentrations containing $15 \%$ Alexa488labeled actin, green.

\section{Cryo-electron microscopy}

340 To prepare small liposomes, a mixture of EPC lipids and $0.1 \%$ biotinylated lipids with a

341 concentration of $1 \mathrm{mg} / \mathrm{mL}$ in chloroform/methanol 5:3 (v/v) is dried and resuspended under

342 vortexing in a buffer containing $25 \mathrm{mM}$ imidazol, $1 \mathrm{mM}$ Tris, $50 \mathrm{mM} \mathrm{KCl,} 2 \mathrm{mM} \mathrm{MgCl} 2,0.1$

$343 \mathrm{mM}$ DTT, $1.6 \mathrm{mM}$ ATP, $0.02 \mathrm{mg} / \mathrm{mL} \beta$-casein. Liposomes are then incubated with SpVCA 
344 (350 nM) for $15 \mathrm{~min}$ and finally flash-frozen for cryo-electron microscopy. Images were recorded under low dose conditions with a Tecnai G2 Lab6 electron microscope operating at $200 \mathrm{kV}$ with a TVIPS F416 4K camera and with a resolution of $0.21 \AA /$ pixel.

\section{Observation of liposomes}

Observation in 2D: epifluorescence (GFP filter cube, excitation $470 \mathrm{~nm}$, emission $525 \mathrm{~nm}$;

Texas red filter cube: excitation $545-580 \mathrm{~nm}$, emission $610 \mathrm{~nm}$-IR), phase contrast and camera (CoolSnap, Photometrics, Roper Scientific).

Observation in 3D: confocal and bright-field microscopy are performed using an inverted (excitation filter: $560-580 \mathrm{~nm} / \mathrm{emission}$ filter: $600-650 \mathrm{~nm}$ ) are used to acquire respectively actin and lipids fluorescence. Images are collected by a charge coupled device CCD camera

360 (CoolSnap HQ2, Photometrics, Roper Scientific). interval of $0.5 \mu \mathrm{m}$.

\section{Image analyses of liposomes, tubes and spikes}

Image analyses are performed with ImageJ software and data are processed on Matlab. The thickness of the actin network and the length of tube membranes is obtained from

367 fluorescence intensity profiles (Fig. 3A). The first peak of the membrane profile determines 368 the liposome surface, the actin network thickness is the distance between this peak and the 
half width at half maximum of the actin fluorescence profile. The length of the membrane tubes is obtained as the peak-to peak distance of the membrane fluorescence profile. The size

371 of spikes (length, width) and actin network is determined by the corresponding positions of

372 the inflexion points. Fluorescence profiles in each case (membrane, actin) are fitted with a

373 polynomial function. The first maximum and the second minimum of the fit derivative,

374 corresponding to inflexion points of the profile, determine the membrane or actin edges. The size is then the distance between the two edges. From actin fluorescence profile, actin network thickness at the base of spike is defined as the distance between the first maximum and first minimum of the fit derivative.

To determine whether shorter tubes are present in addition to the easily visualized long ones, we measure the total fluorescence intensity of the membrane on an arc that is displaced along a radial axis $r$ from close to the liposome surface to the external part of the network. We hypothesize that tubes maintain a constant diameter along their length, as is established for pure membrane tubes ${ }^{19}$. In these conditions, if all tubes have the same length, the total intensity should show a plateau as a function of $r$, until falling off to zero at an $r$ where there are no more tubes (Supplementary Fig. 4A). Conversely, the total intensity would decrease as a function of $r$ if tubes of different lengths were present (Supplementary Fig. 4A).

\section{Statistical analyses}

388 All statistical analyses are performed using MedCalc software. N-1 Chi-squared test is used

389 to determine the statistical significance. Differences among samples were considered

390 statistically significant when $\mathrm{p}<0.05$. 
393 To calculate the stress exerted by a viscous network, polymerizing at a curved surface we

394 consider an incompressible stokes flow, described by force balance and incompressibility,

395 i.e., $\vec{\nabla} \cdot \vec{\sigma}=0$ and $\vec{\nabla} \cdot \vec{v}=0$, where $\vec{v}$ is the velocity of the gel and $\vec{\sigma}$ is the viscous stress in

396 Cartesian coordinates, given by, $\sigma_{i j}=-p \delta_{i j}+\eta\left(\frac{\partial v_{i}}{\partial x_{j}}+\frac{\partial v_{j}}{\partial x_{i}}\right)$. Polymerization of the actin

397 network is encoded in this model by imposing the velocity of the network, normal to the

398 surface of the curved interface. Moreover, we impose a stress free boundary condition at the

399 outer layer, both for the normal as well as the tangential stress, i.e., $\sigma_{n n}=0$ and $\sigma_{n t}=0$.

400 Note that, in the limit we consider, an infinite thick network, this corresponds to a uniform

401 velocity in the z-direction.

402

403 We determine the first order correction of the normal stress on a deformed surface

404 characterized by $u(x)=u_{0} \exp (i q x)$ along the $x$ axis $\left(u_{0}\right.$ is the deformation amplitude and $q$

405 the wave vector, Fig. 5A). We seek a solution for the velocity field within the network of the

406 form $v_{j}=v_{j}(z) \exp (i q x)$, where the index $j$ represents the coordinate $x$ or $z$, and a pressure

407 field of the form $p=p(z) \exp (i q x)$. Assuming that the network grows normal to the surface,

408 the first order correction of the $x$-component of the velocity field satisfies the boundary

409 condition $\delta v_{x}(z=0)=-v_{p} \partial_{x} u(x)$ at the interface $(z=0)$. We assume here a network of

410 large thickness and require that the first order correction to the velocity vanishes at $z \rightarrow \infty$.

411 The first order corrections to the velocity and pressure in the network read $\delta v_{x}(z)=$

$412-i q u_{0}(1-q z) v_{p} \exp (-q z), \delta v_{z}(z)=-q^{2} u_{0} v_{p} z \exp (-q z)$ and $\delta p(z)=$

$413-2 \eta q^{2} u_{0} v_{p} \exp (-q z)$. At this order the actin normal stress turns out to vanish at any point

414 of the liposome surface: $\sigma_{n n}(x, z=0)=2 \eta \partial_{z} v_{z}-p=0$. This implies that the membrane is

415 linearly stable against small deformations in the presence of a growing actin network. 
416 The second-order correction for the actin stress is in principle difficult to calculate, as the

417 different modes of deformation are coupled. An analytical estimate can be obtained by

418 expanding the surface normal vector up to second order, which yields the following scaling

419 for the normal stress at the liposome surface, $\sigma_{n n} \propto-\eta q^{3} u_{0}^{2} v_{p}$. This weakly non-linear

420 analysis reveals that there is a non-zero normal stress acting on the membrane, which we will

421 later compare with the membrane contribution to address system stability.

422 In order to get a numerical solution for the normal stress in a "localized" spike-like

423 perturbation on the interface, as opposed to the periodic one presented above, we use a Finite

424 Element Method from Mathematica with default settings. We implement a geometry as

425 described in Fig. 5B, where the lower surface is parametrized with a Gaussian deformation as

426 mentioned before, i.e, $u(x, z)=z-A \exp -\left(\frac{x}{b}\right)^{2}=0$ and we choose the height of the

427 system to be much larger that the extend and amplitude of the perturbation $(h=2 \mu m)$. Note

428 that here, $b$, the characteristic lateral length of the localized perturbation, is related to the

429 wavenumber $q \sim 1 / b$ used for the linear analysis. To account for a constant polymerization,

430 perpendicular to the lower surface we impose the velocity on the lower surface, i.e.,

$\partial v(u(x, z)=0)=v_{p}\left(\partial_{x} u(x, z) \hat{x}+\partial_{z} u(x, z) \hat{z}\right)$, where $v_{p}$ is the normalized polymerization

432 velocity and a vanishing normal and tangential stress at the upper boundary $z=h$,

433 i.e., $\sigma_{n n}(z=h)=0$ and $\sigma_{n t}(z=h)=0$. Using this approach we could find the same

434 scaling with amplitude and width of the perturbation, as found for the weakly non-linear

435 analysis for a sinusoidal perturbation. Note also that here, by imposing the normal velocity at

436 the interface, a choice that is motivated by the dual color images in Fig. 4A, we do not

437 impose the tangential stress on the membrane, and hence this stress has to be balanced by an

438 in-plane viscous stress in the membrane, which at this stage we do not model. These FEM

439 simulations allow us to visualize the velocity field as well as the pressure throughout the 
440 network, indicating the increase in pressure inside the local perturbation caused by the local 441 convergence of the velocity fields (Fig. 5C).

442

\section{References}

4441 Kukulski, W., Schorb, M., Kaksonen, M. \& Briggs, J. A. Plasma membrane reshaping 445 during endocytosis is revealed by time-resolved electron tomography. Cell 150, 508$446 \quad$ 520, doi:10.1016/j.cell.2012.05.046 (2012).

4472 Picco, A., Mund, M., Ries, J., Nedelec, F. \& Kaksonen, M. Visualizing the functional $448 \quad$ architecture of the endocytic machinery. Elife 4, doi:10.7554/eLife.04535 (2015).

4493 Picco, A. et al. The contributions of the actin machinery to endocytic membrane $450 \quad$ bending and vesicle formation. bioRxiv, doi:10.1101/172072 (2017).

4514 Boulant, S., Kural, C., Zeeh, J. C., Ubelmann, F. \& Kirchhausen, T. Actin dynamics 452 counteract membrane tension during clathrin-mediated endocytosis. Nat Cell Biol 13, 1124-1131, doi:10.1038/ncb2307 (2011).

4545 Korobova, F. \& Svitkina, T. Molecular architecture of synaptic actin cytoskeleton in 455 hippocampal neurons reveals a mechanism of dendritic spine morphogenesis. Mol 456 Biol Cell 21, 165-176, doi:10.1091/mbc.E09-07-0596 (2010).

4576 van der Gucht, J., Paluch, E., Plastino, J. \& Sykes, C. Stress release drives symmetry 458 breaking for actin-based movement. Proc Natl Acad Sci U S A 102, 7847-7852 $459 \quad$ (2005).

4607 Carvalho, K. et al. Actin polymerization or myosin contraction: two ways to build up 461 cortical tension for symmetry breaking. Philos Trans R Soc Lond B Biol Sci 368, 20130005, doi:10.1098/rstb.2013.0005 (2013).

4638 Paluch, E., Piel, M., Prost, J., Bornens, M. \& Sykes, C. Cortical actomyosin breakage $464 \quad$ triggers shape oscillations in cells and cell fragments. Biophys $J$ 89, 724-733 (2005). 
4659 Akin, O. \& Mullins, R. D. Capping protein increases the rate of actin-based motility

466 by promoting filament nucleation by the Arp2/3 complex. Cell 133, 841-851,

467 doi:10.1016/j.cell.2008.04.011 (2008).

$46810 \quad$ Julicher, F., Kruse, K., Prost, J. \& Joanny, J. F. Active behavior of the cytoskeleton.

$469 \quad$ Physics reports 449, 3-28 (2007).

47011 Gardel, M. L. et al. Scaling of F-actin network rheology to probe single filament $471 \quad$ elasticity and dynamics. Phys Rev Lett 93, 188102,

472 doi:10.1103/PhysRevLett.93.188102 (2004).

47312 Gardel, M. L., Kasza, K. E., Brangwynne, C. P., Liu, J. \& Weitz, D. A. Chapter 19:

474 Mechanical response of cytoskeletal networks. Methods Cell Biol 89, 487-519, 475 doi:10.1016/s0091-679x(08)00619-5 (2008).

47613 Noireaux, V. et al. Growing an actin gel on spherical surfaces. Biophys J 78, 1643$4771654(2000)$.

47814 Mogilner, A. \& Rubinstein, B. The physics of filopodial protrusion. Biophys J 89, 479 782-795, doi:10.1529/biophysj.104.056515 (2005).

48015 Prost, J., Barbetta, C. \& Joanny, J. F. Dynamical control of the shape and size of $481 \quad$ stereocilia and microvilli. Biophys J 93, 1124-1133, doi:10.1529/biophysj.106.098038 $482 \quad$ (2007).

48316 Caorsi, V. et al. Cell-sized liposome doublets reveal active tension build-up driven by 484 acto-myosin dynamics. Soft Matter 12, 6223-6231, doi:10.1039/c6sm00856a (2016). 48517 Marcy, Y., Prost, J., Carlier, M.-F. \& Sykes, C. Forces generated during actin-based 486 propulsion: a direct measurement by micromanipulation. Proc. Natl. Acad. Sci. USA $487 \quad \mathbf{1 0 1}, 5993-5997(2004)$.

48818 Deserno, M. Fluid lipid membranes: from differential geometry to curvature stresses. 489 Chem Phys Lipids 185, 11-45, doi:10.1016/j.chemphyslip.2014.05.001 (2015). 
49019 Derenyi, I., Julicher, F. \& Prost, J. Formation and interaction of membrane tubes.

$491 \quad$ Phys Rev Lett 88, 238101, doi:10.1103/PhysRevLett.88.238101 (2002).

49220 Roux, A. et al. Role of curvature and phase transition in lipid sorting and fission of 493 membrane tubules. Embo J 24, 1537-1545, doi:10.1038/sj.emboj.7600631 (2005).

49421 Smith, B. A. et al. Three-color single molecule imaging shows WASP detachment 495 from Arp2/3 complex triggers actin filament branch formation. Elife 2, e01008, doi:10.7554/eLife.01008 (2013).

49722 Kroy, K. \& Frey, E. Force-Extension Relation and Plateau Modulus for Wormlike Chains. Phys Rev Lett 77, 306-309, doi:10.1103/PhysRevLett.77.306 (1996).

49923 Kawska, A. et al. How actin network dynamics control the onset of actin-based $500 \quad$ motility. Proc Natl Acad Sci U S A 109, 14440-14445, doi:10.1073/pnas.1117096109 501 (2012).

50224 Wang, X. \& Carlsson, A. E. A master equation approach to actin polymerization applied to endocytosis in yeast. PLoS Comput Biol 13, e1005901, doi:10.1371/journal.pcbi.1005901 (2017).

50525 Carlsson, A. E. Membrane bending by actin polymerization. Curr Opin Cell Biol 50, 506 1-7, doi:10.1016/j.ceb.2017.11.007 (2017).

$50726 \quad$ Aghamohammadzadeh, S. \& Ayscough, K. R. Differential requirements for actin 508 during yeast and mammalian endocytosis. Nat Cell Biol 11, 1039-1042, 509 doi:10.1038/ncb1918 (2009).

51027 Dmitrieff, S. \& Nedelec, F. Membrane Mechanics of Endocytosis in Cells with 511 Turgor. PLoS Comput Biol 11, e1004538, doi:10.1371/journal.pcbi.1004538 (2015).

51228 Carlsson, A. E. \& Bayly, P. V. Force generation by endocytic actin patches in budding yeast. Biophys J 106, 1596-1606, doi:10.1016/j.bpj.2014.02.035 (2014). 
51429 Sun, Y. et al. Switch-like Arp2/3 activation upon WASP and WIP recruitment to an

515 apparent threshold level by multivalent linker proteins in vivo. Elife 6,

516 doi:10.7554/eLife.29140 (2017).

$51730 \quad$ Lan, Y. \& Papoian, G. A. The stochastic dynamics of filopodial growth. Biophys J 94,

$518 \quad$ 3839-3852, doi:10.1529/biophysj.107.123778 (2008).

$51931 \quad$ Atilgan, E., Wirtz, D. \& Sun, S. X. Mechanics and dynamics of actin-driven thin

520 membrane protrusions. Biophys J 90, 65-76, doi:10.1529/biophysj.105.071480

521 (2006).

52232 Liu, A. P. et al. Membrane induced bundling of actin filaments. Nature Physics 4, 789-793 (2008).

52433 Hotulainen, P. et al. Defining mechanisms of actin polymerization and depolymerization during dendritic spine morphogenesis. The Journal of Cell Biology 185, 323 (2009).

34 Palmgren, S., Ojala, P. J., Wear, M. A., Cooper, J. A. \& Lappalainen, P. Interactions with PIP2, ADP-actin monomers, and capping protein regulate the activity and localization of yeast twinfilin. J Cell Biol 155, 251-260, doi:10.1083/jcb.200106157

530 (2001).

35 Havrylenko, S. et al. WAVE binds Ena/VASP for enhanced Arp2/3 complex-based actin assembly. Molecular Biology of the Cell 26, 55-65, doi:10.1091/mbc.E14-071200 (2015).

\section{Acknowledgments}

536 We acknowledge Dr. Agnieszka Kawska at IlluScientia.com for the Fig.s. This work was

537 supported by the French Agence Nationale pour la Recherche (ANR), grant ANR

538 09BLAN0283 and ANR 12BSV5001401, by Fondation pour la Recherche Médicale (FRM), 
539 grant DEQ20120323737, by the LabEx CelTisPhyBio postdoctoral fellowship (ML), No.

540 ANR-10-LBX-0038 part of the IDEX PSL NANR-10-IDEX-0001-02 PSL, by Marie Curie

541 Integration Grant PCIG12-GA-2012-334053, “Investissements d'Avenir” LabEx PALM

542 (ANR-10-LABX-0039-PALM), ANR grant ANR-15-CE13-0004-03 and ERC Starting Grant

543 677532. Our groups belong to the CNRS consortium CellTiss. This work was supported by

544 grants from the French National Research Agency through the "Investments for the Future"

545 (France-BioImaging, ANR-10-INSB-04), the PICT-IBiSA Institut Curie (Paris, France)

547 Author contributions: CS, RK and VC have equal contributions. CS, VC performed

548 experiments, analyzed data. RK performed the development of theoretical models. AA and

549 MAG, JM, AdC, DL, CC, JP contributed to experimental data, ML and JFJ contributed to the

550 development of the model, PS and CS designed the research. All authors contributed to write

551 the paper.

552

553 Author information: request for material should be addressed to PS and CS

554 cecile.sykes@curie.fr. 
A

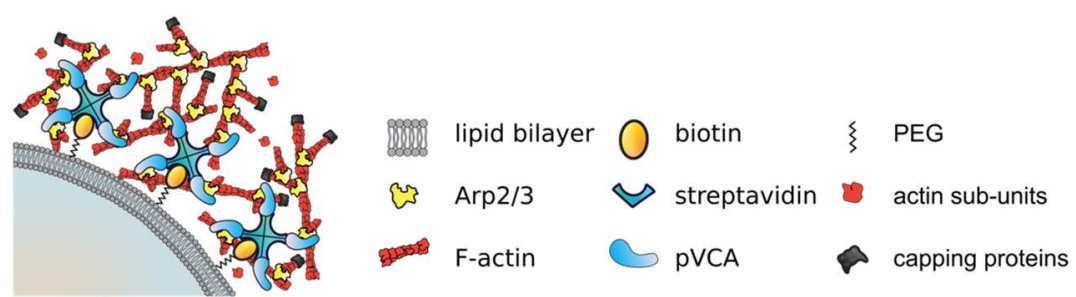

B

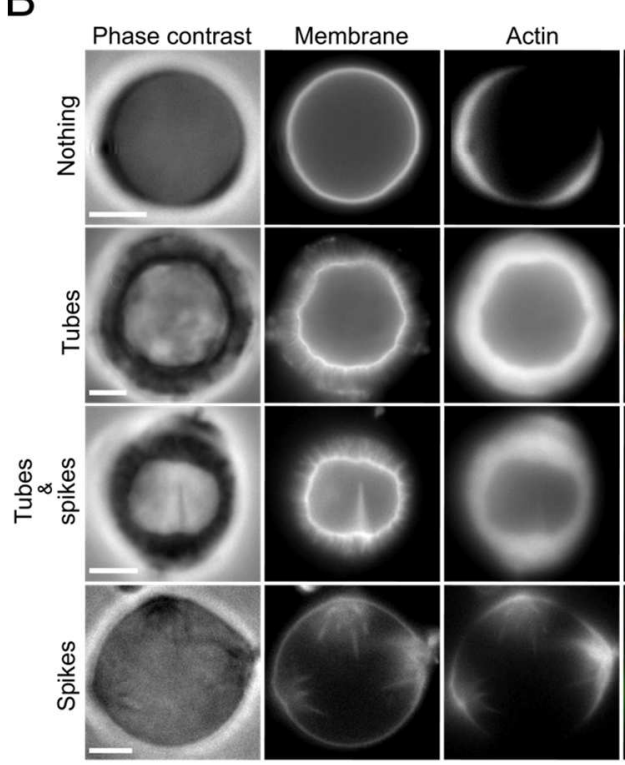

C

D
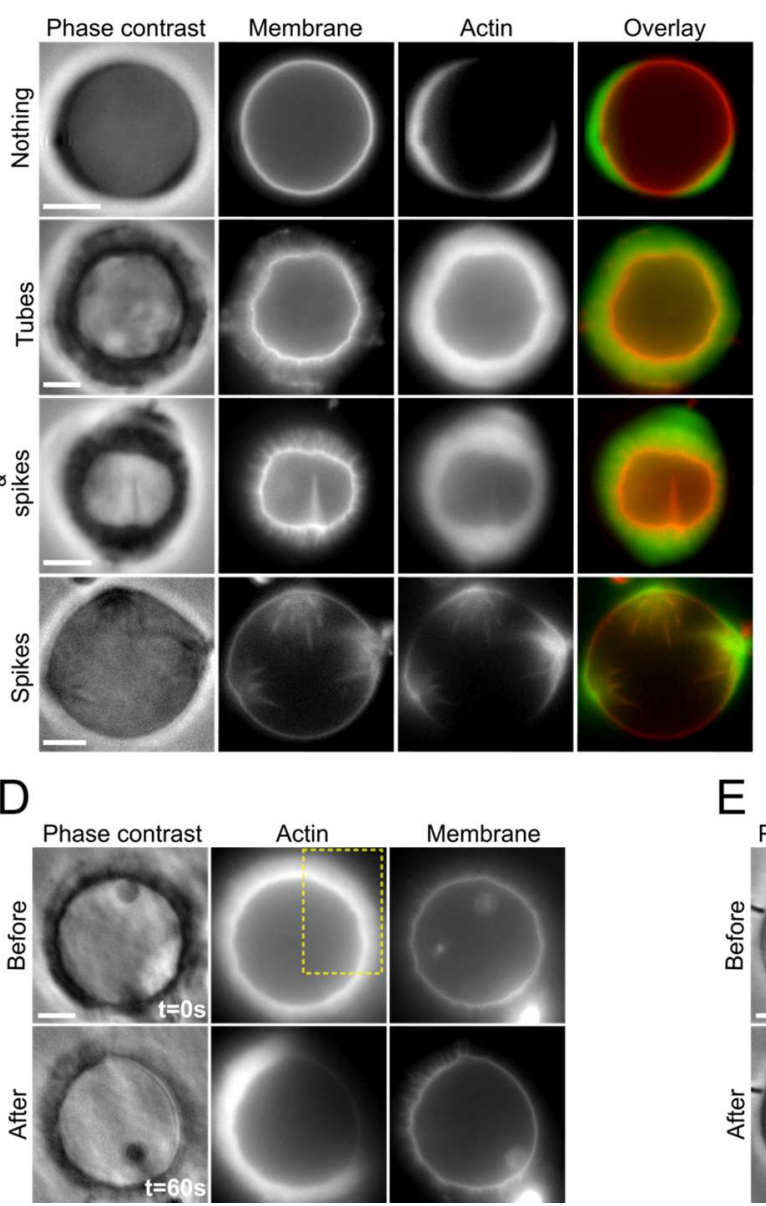

Non- deflated Added sucrose Deflated liposome Added sucrose liposome
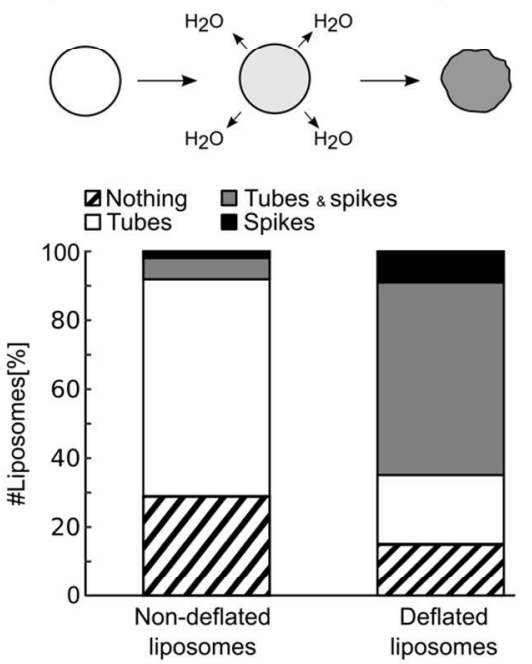

Figure 1: Experimental system and observations

E

557 (A) Scheme of the experimental system; proteins not to scale. (B) Membrane deformations in both non-deflated (three first rows) and deflated conditions (last row).

(C) Top: liposome

559 deflation. Bottom: number of liposomes displaying nothing, tubes, both tubes and spikes, and

560 only spikes. Non-deflated liposomes, $n=311$. Deflated liposomes, $n=123$. (D, E) Actin

561 network photo-damage (yellow dashed rectangle) on a liposome displaying membrane tubes

562 (D) or spikes (E). Phase contrast and epifluorescence microscopy of membrane and actin

563 network. Scale bars, $5 \mu \mathrm{m}$. 


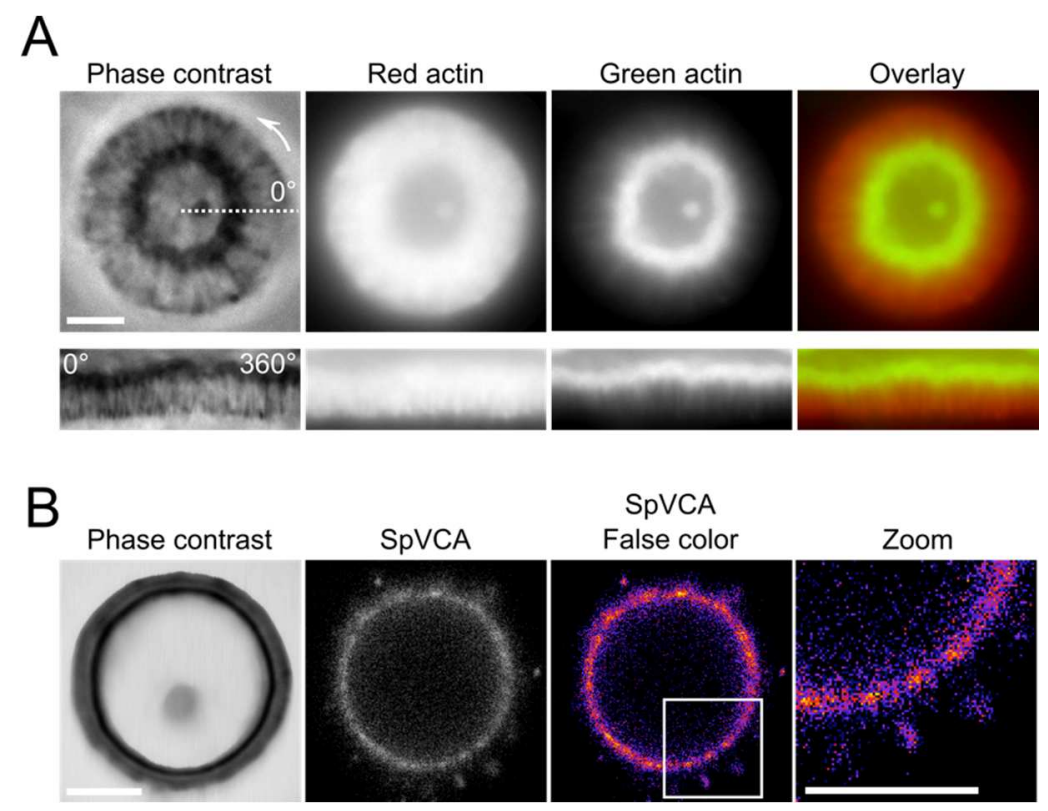

564 Figure 2: Actin incorporation during tube formation

565 (A) Top: a red actin network is grown for 20 minutes, then an excess of green actin is added,

566 so green regions indicate newly polymerized actin. Bottom: corresponding polar plots. (B)

567 Images of the activator of actin polymerization, SpVCA. False color image and zoom in

568 (white rectangle). Phase contrast and epifluorescence microscopy of the actin network

569 labeled with actin-Alexa568 (red) and actin-Alexa488 (green) in (A), and of SpVCA-

$570 \quad$ Alexa546 in (B). Scale bars, $5 \mu \mathrm{m}$. 
A

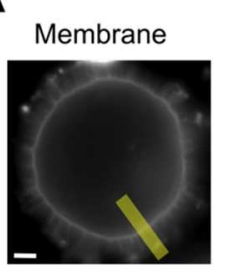

Actin

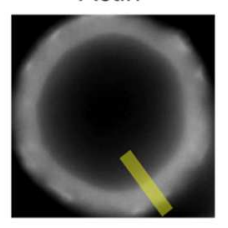

C

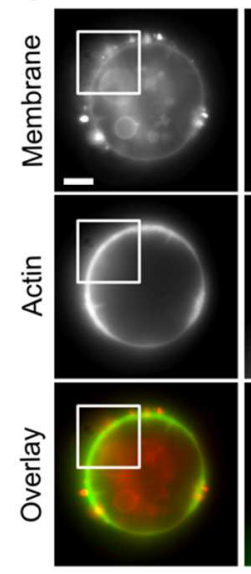

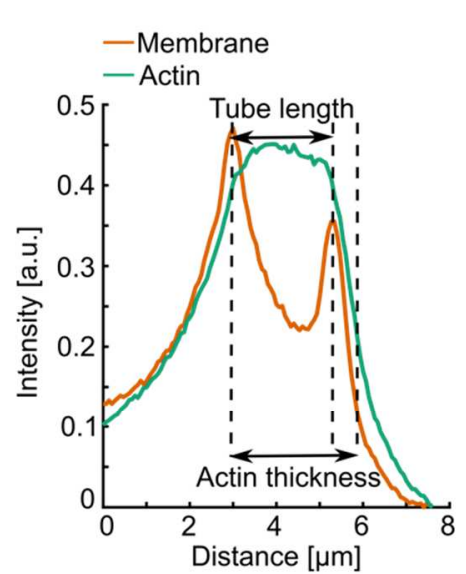

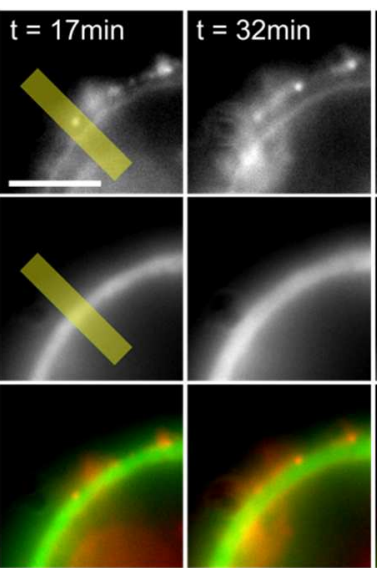

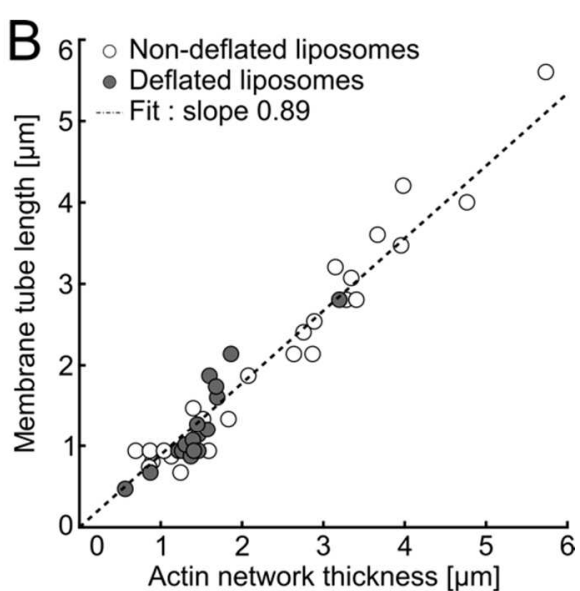

D - Membrane

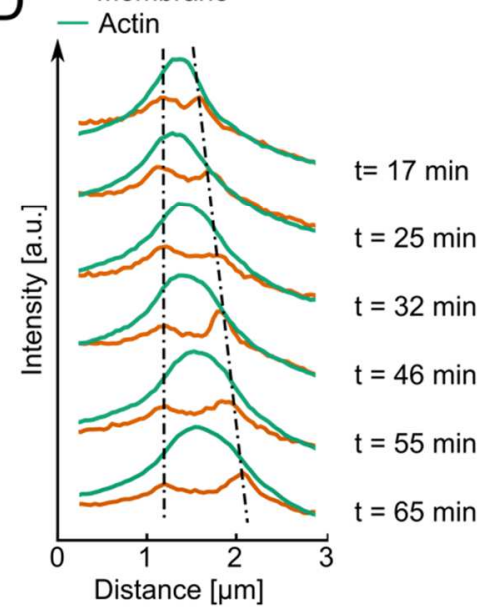

$571 \quad$ Figure 3: Tube length compared to network thickness

572 (A) Tube length and actin network thickness are measured from fluorescence intensity

573 profiles (thick yellow line) of the membrane (red) and the actin (green) channels (Materials

574 and Methods). (B) Tube length as a function of actin network thickness. White circles: non-

575 deflated liposomes. Grey circles: deflated liposomes. (C) Dynamics of tube growth (times

576 indicate elapsed time from the start of actin polymerization). (D) Fluorescence profile of the

577 thick yellow lines shown in (C). Membrane and actin fluorescence intensities plotted over

578 time (indicated). Other examples are shown in Supplementary Fig. 2. Epifluorescence

579 microscopy of membrane and actin. Scale bars, $5 \mu \mathrm{m}$. 
A
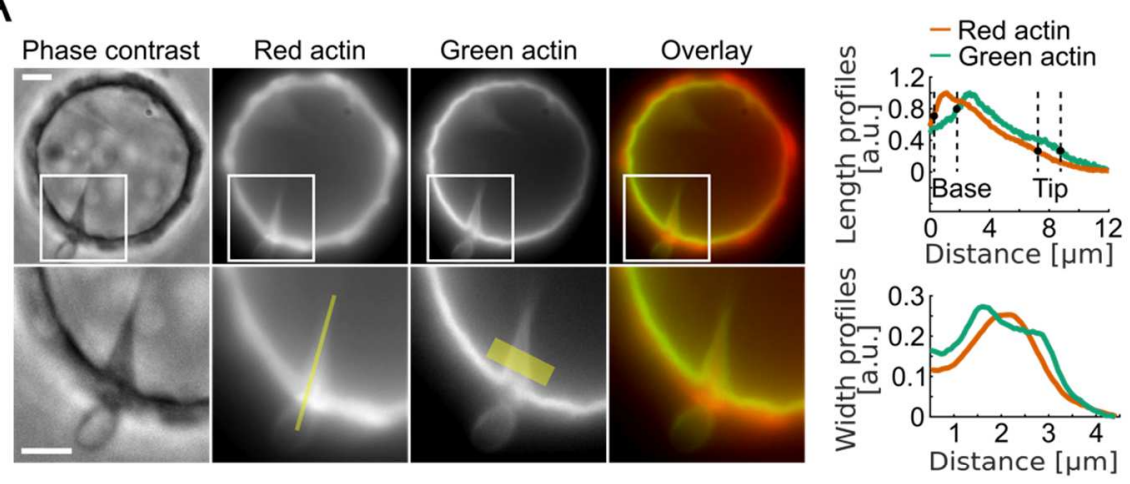

B

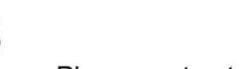

SpVCA

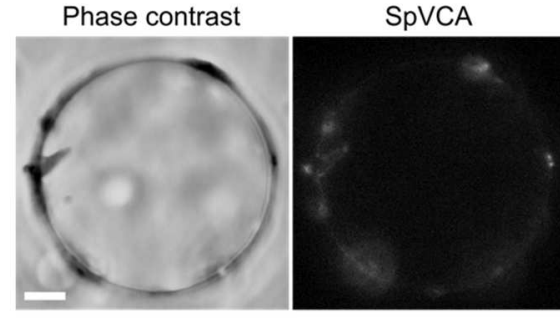

False color

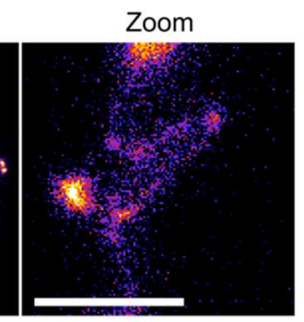

C
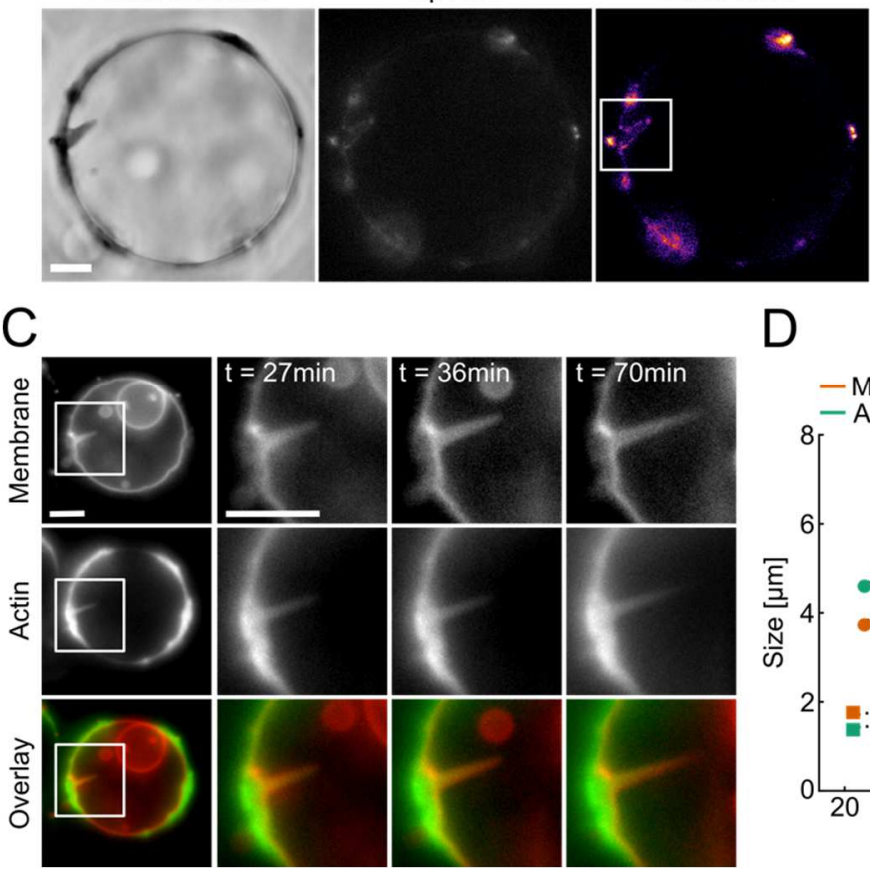

Figure 4: Actin incorporation in spikes

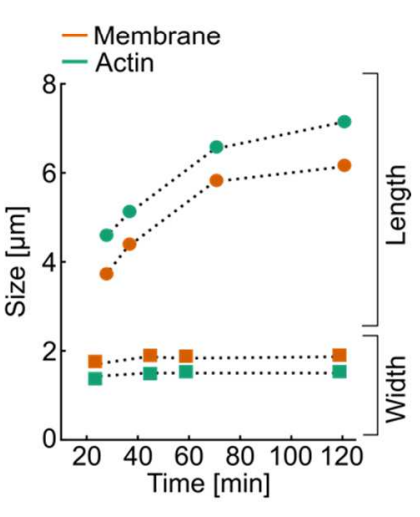

581 (A) Left: Two color experiment: green regions indicate newly polymerized actin. White

582 squares, zooms. Right: fluorescence intensity profiles spike length (top, thin yellow line on

583 zoomed image) and width (bottom, thick yellow line on zoomed image). (B) Activator of

584 actin polymerization, SpVCA. White rectangle, zoom. (C) Dynamics of spike growth (time

585 after actin polymerization starts). (D) Spike length and width over time, spike shown in C.

586 Other examples in Supplementary Fig. 5. Dashed lines, guides to the eyes. Phase contrast and

587 epifluorescence microscopy of the actin network (A), SpVCA-Alexa546 (B) membrane and actin network (C). Scale bars, $5 \mu \mathrm{m}$. 
A

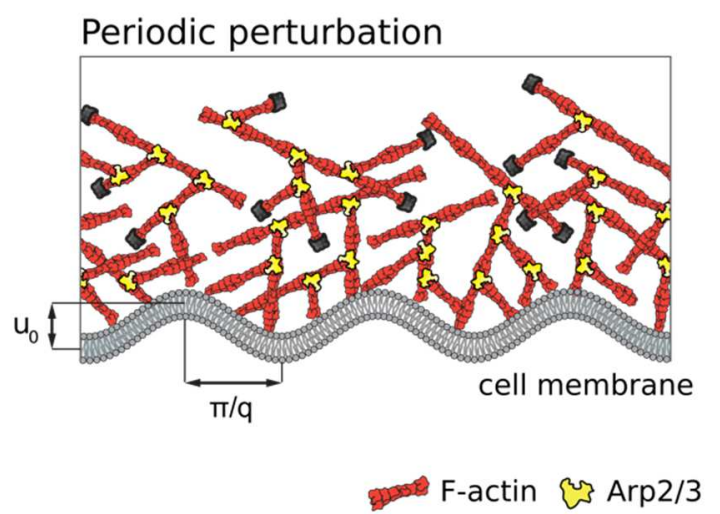

C

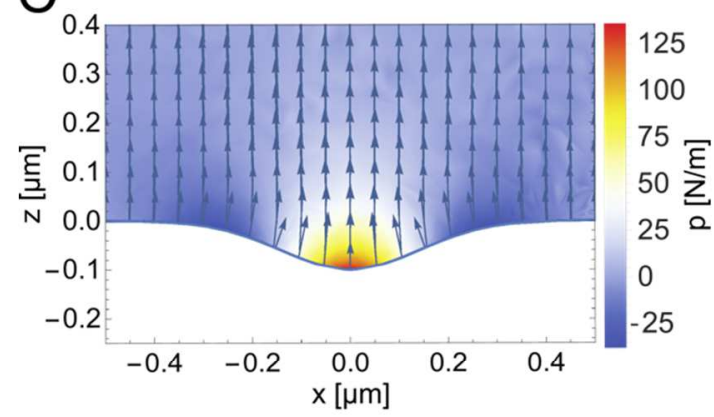

$E$

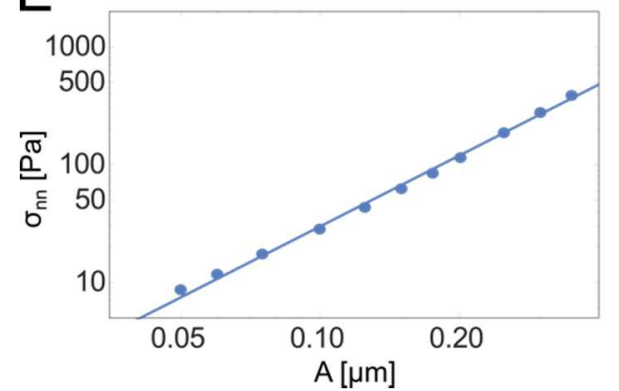

B

Localized perturbation

capping proteins

D

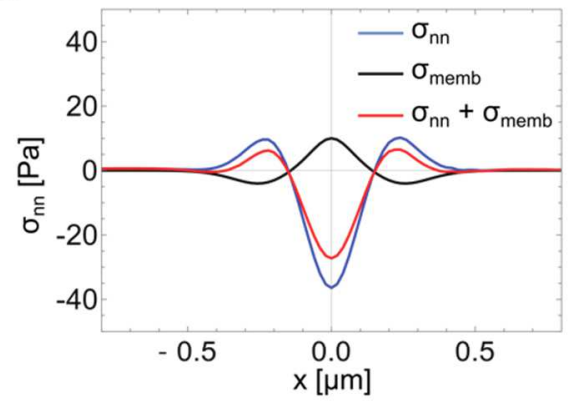

$\mathrm{F}$

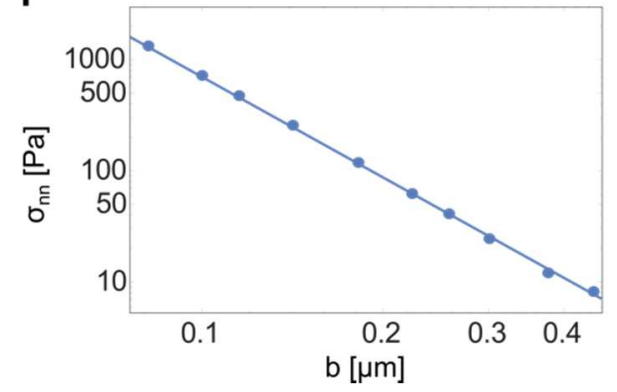

589 Figure 5: Model for spike initiation

590 Scheme of the initiation of a periodic (A) and localized (B) membrane deformation by the

591 growth of the actin network. (C) Velocity field of a viscous network polymerizing over a

592 membrane with a localized (gaussian) perturbation (amplitude $A=0.1 \mu \mathrm{m}$, width $b=0.2 \mu \mathrm{m}$,

593 polymerization velocity $v_{p}=1 \mathrm{~nm} / \mathrm{s}$, viscosity $\eta=10^{4} \mathrm{~Pa} . \mathrm{s}$ ). Color, pressure in the network

594 layer. (D) Corresponding distribution of actin and membrane normal stresses ( $\sigma_{n n}$ and $\sigma_{m e m b}$

595 respectively). (E, F) Scaling of $\sigma_{n n}$ as function of the amplitude for a value of $b=0.22 \mu \mathrm{m}$

596 (E) and width for a value of $A=0.15 \mu \mathrm{m}$ (F) of the perturbation $\left(v_{p}=1 \mathrm{~nm} / \mathrm{s}\right.$, viscosity $\eta=10^{4}$

597 Pa.s). 


\section{A}

Initial attachment

Growth

Retraction

Stabilization
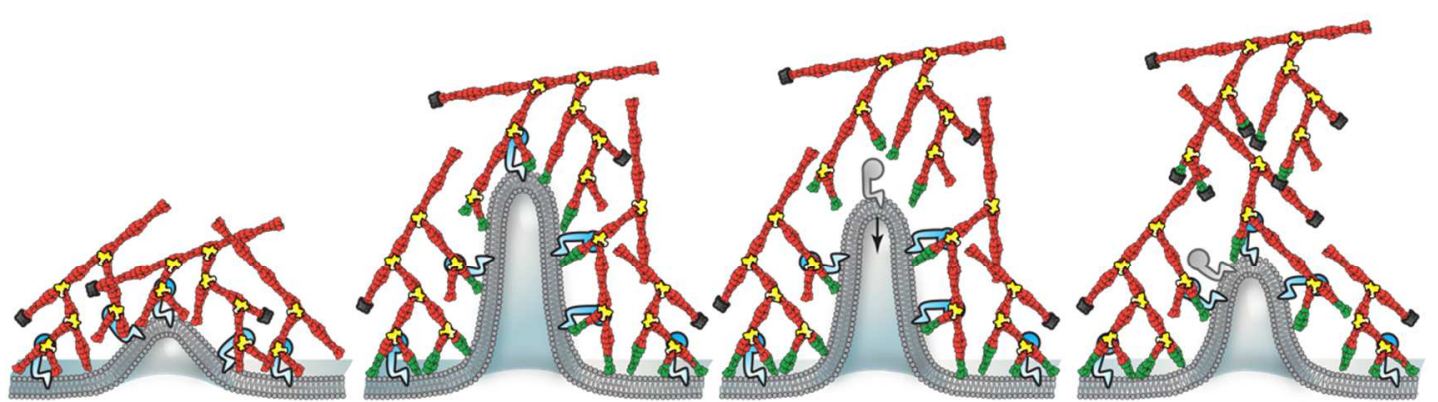

cell membrane

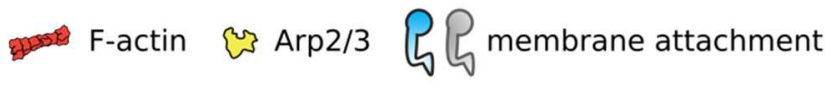

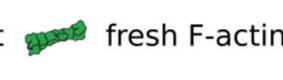

capping proteins

B

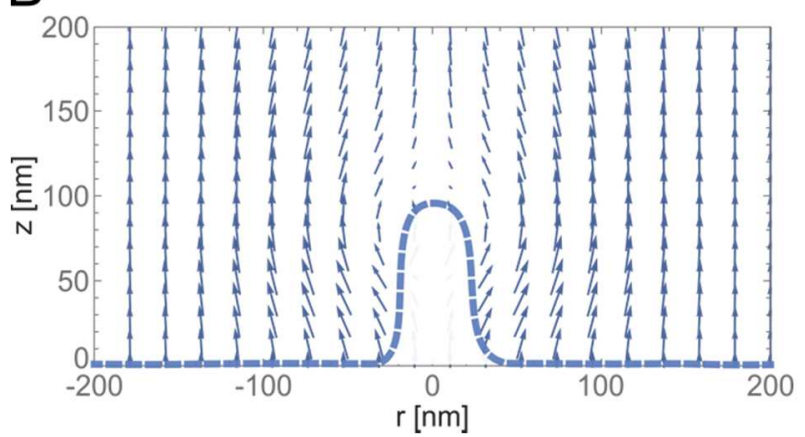

C

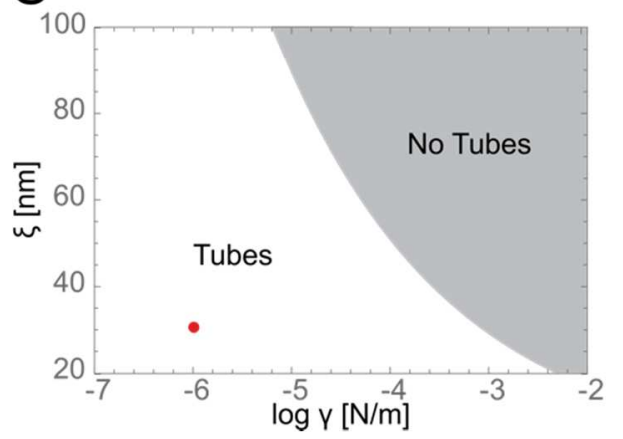

598

\section{Figure 6: Model for tube initiation}

599 (A) Scheme of a membrane tube pulled by the actin network and retraction due to

600 detachment. (B) Velocity field of the actin network pulling the membrane tube. We assume a

601 uniform polymerization $v_{p}$ at the liposome surface and model the presence of the tube as a

602 disc with radius $r_{\text {tube }}=20 \mathrm{~nm}$ and height $h=100 \mathrm{~nm}$. (C) Phase diagram, mechanics of

603 tube pulling as a function of mesh size $\xi$ and membrane tension $\gamma$. Grey part, region where

604 the viscous driving force is not sufficient to extract a tube $\left(f_{\text {tube }}=2 \mathrm{pN}, \kappa=10 \mathrm{kT}\right.$ and $h=$

$605140 \mathrm{~nm}$ ). Red point, our experimental conditions. 